\title{
On Parametric $S$-Metric Spaces and Fixed-Point Type Theorems for Expansive Mappings
}

\author{
Nihal Taş and Nihal Yılmaz Özgür \\ Department of Mathematics, Ballkesir University, 10145 Balıesir, Turkey \\ Correspondence should be addressed to Nihal Taş; nihaltas@balikesir.edu.tr \\ Received 29 July 2016; Revised 11 October 2016; Accepted 16 October 2016 \\ Academic Editor: Kaleem R. Kazmi
}

Copyright (C) 2016 N. Taş and N. Yılmaz Özgür. This is an open access article distributed under the Creative Commons Attribution License, which permits unrestricted use, distribution, and reproduction in any medium, provided the original work is properly cited.

\begin{abstract}
We introduce the notion of a parametric $S$-metric space as generalization of a parametric metric space. Using some expansive mappings, we prove a fixed-point theorem on a parametric $S$-metric space. It is important to obtain new fixed-point theorems on a parametric $S$-metric space because there exist some parametric $S$-metrics which are not generated by any parametric metric. We expect that many mathematicians will study various fixed-point theorems using new expansive mappings (or contractive mappings) in a parametric $S$-metric space.
\end{abstract}

\section{Introduction and Backgrounds}

Contractive conditions have been started by studying Banach's contraction principle. These conditions have been used in various fixed-point theorems for some generalized metric spaces. Then expansive conditions were introduced [1] and new fixed-point results were obtained using expansive mappings.

Recently, the notion of an S-metric has been studied by some mathematicians. This notion was introduced by Sedghi et al. in 2012 [2] as follows.

Definition 1 (see [2]). Let $X$ be a nonempty set and let $S$ : $X \times X \times X \rightarrow[0, \infty)$ be a function. $S$ is called an $S$-metric on $X$ if,

$$
\begin{aligned}
& \text { (S1) } S(a, b, c)=0 \text { if and only if } a=b=c, \\
& \text { (S2) } S(a, b, c) \leq S(a, a, x)+S(b, b, x)+S(c, c, x),
\end{aligned}
$$

for each $a, b, c, x \in X$. The pair $(X, S)$ is called an $S$-metric space.

Using the notion of an $S$-metric space, various meaningful fixed-point studies were obtained by some researchers (see [2-6] for more details).
The relationship between a metric and an $S$-metric was studied and an example of an $S$-metric which is not generated by any metric was given in $[3,4]$.

Later, the notion of a parametric metric space was introduced and some basic concepts such as a convergent sequence and a Cauchy sequence were defined in [7]. We recall the following definitions.

Definition 2 (see [7]). Let $X$ be a nonempty set and let $P$ : $X \times X \times(0, \infty) \rightarrow[0, \infty)$ be a function. $P$ is called a parametric metric on $X$ if,

$$
\begin{aligned}
& \text { (P1) } P(a, b, t)=0 \text { if and only if } a=b \text {, } \\
& \text { (P2) } P(a, b, t)=P(b, a, t), \\
& \text { (P3) } P(a, b, t) \leq P(a, x, t)+P(x, b, t),
\end{aligned}
$$

for each $a, b, x \in X$ and all $t>0$. The pair $(X, P)$ is called a parametric metric space.

Definition 3 (see [7]). Let $(X, P)$ be a parametric metric space and let $\left\{a_{n}\right\}$ be a sequence in $X$ :

(1) $\left\{a_{n}\right\}$ converges to $x$ if and only if there exists $n_{0} \in \mathbb{N}$ such that

$$
P\left(a_{n}, x, t\right)<\varepsilon
$$


for all $n \geq n_{0}$ and all $t>0$; that is,

$$
\lim _{n \rightarrow \infty} P\left(a_{n}, x, t\right)=0 .
$$

It is denoted by $\lim _{n \rightarrow \infty} a_{n}=x$.

(2) $\left\{a_{n}\right\}$ is called a Cauchy sequence if, for all $t>0$,

$$
\lim _{n, m \rightarrow \infty} P\left(a_{n}, a_{m}, t\right)=0 .
$$

(3) $(X, P)$ is called complete if every Cauchy sequence is convergent.

In the following definition, the concept of a parametric $b$-metric space as generalization of a parametric metric space was given.

Definition 4 (see [8]). Let $X$ be a nonempty set, let $s \geq 1$ be a real number, and let $P: X \times X \times(0, \infty) \rightarrow[0, \infty)$ be a function. $P$ is called a parametric $b$-metric on $X$ if,

$$
\begin{aligned}
& \text { (Pb1) } P(a, b, t)=0 \text { if and only if } a=b, \\
& (P b 2) P(a, b, t)=P(b, a, t), \\
& (P b 3) P(a, b, t) \leq s[P(a, x, t)+P(x, b, t)],
\end{aligned}
$$

for each $a, b, x \in X$ and all $t>0$. The pair $(X, P)$ is called a parametric $b$-metric space.

Notice that a parametric $b$-metric is sometimes called a parametric $s$-metric according to a real number $s \geq 1$ in the above definition (see [9]).

Some fixed-point theorems have been still investigated using the notions of a parametric metric space and a parametric $b$-metric space for various contractive or expansive mappings (see [7-10] for more details). For example, Hussain et al. proved some fixed-point theorems on complete parametric metric spaces and triangular intuitionistic fuzzy metric spaces [7]. Also, Hussain et al. introduced the notion of parametric $b$-metric space and investigated some fixedpoint results [8]. Jain et al. established some fixed-point, common fixed-point, and coincidence point theorems for expansive type mappings on parametric metric spaces and parametric $b$-metric spaces [10]. Rao et al. obtained two common fixed-point theorems on parametric $s$-metric spaces [9].

The aim of this paper is to introduce the concept of a parametric $S$-metric and give some basic facts. We give two examples of a parametric $S$-metric which is not generated by any parametric metric. We prove some fixed-point results under various expansive mappings in a parametric $S$-metric space. Also, we verify our results with some examples.

\section{Parametric $S$-Metric Spaces}

In this section, we introduce the notion of "a parametric $S$ metric space" and give some basic properties of this space. Also, we investigate a relationship between a parametric metric and a parametric $S$-metric (resp., a parametric $b$ metric and a parametric $S$-metric).
Definition 5. Let $X$ be a nonempty set and let $P_{S}: X \times X \times$ $X \times(0, \infty) \rightarrow[0, \infty)$ be a function. $P_{S}$ is called a parametric $S$-metric on $X$ if,

$$
\begin{aligned}
& (P S 1) P_{S}(a, b, c, t)=0 \text { if and only if } a=b=c, \\
& (P S 2) P_{S}(a, b, c, t) \leq P_{S}(a, a, x, t)+P_{S}(b, b, x, t)+ \\
& P_{S}(c, c, x, t),
\end{aligned}
$$

for each $a, b, c \in X$ and all $t>0$. The pair $\left(X, P_{S}\right)$ is called a parametric $S$-metric space.

Now we give the following examples of parametric $S$ metric spaces.

Example 6. Let $X=\{f \mid f:(0, \infty) \rightarrow \mathbb{R}$ be a function $\}$ and let the function $P_{S}: X \times X \times X \times(0, \infty) \rightarrow[0, \infty)$ be defined by

$$
P_{S}(f, g, h, t)=|f(t)-h(t)|+|g(t)-h(t)|,
$$

for each $f, g, h \in X$ and all $t>0$. Then $P_{S}$ is a parametric $S$-metric and the pair $\left(X, P_{S}\right)$ is a parametric $S$-metric space.

Example 7. Let $X=\mathbb{R}$ and let the function $P_{S}: X \times X \times X \times$ $(0, \infty) \rightarrow[0, \infty)$ be defined by

$$
P_{S}(a, b, c, t)=g(t)(|a-b|+|b-c|+|a-c|),
$$

for each $a, b, c \in \mathbb{R}$ and all $t>0$, where $g:(0, \infty) \rightarrow(0, \infty)$ is a continuous function. Then $P_{S}$ is a parametric $S$-metric and the pair $\left(\mathbb{R}, P_{S}\right)$ is a parametric $S$-metric space.

Example 8. Let $X=\mathbb{R}^{+} \cup\{0\}$ and let the function $P_{S}: X \times$ $X \times X \times(0, \infty) \rightarrow[0, \infty)$ be defined by

$$
P_{S}(a, b, c, t)= \begin{cases}0 ; & \text { if } a=b=c, \\ g(t) \max \{a, b, c\} ; & \text { otherwise }\end{cases}
$$

for each $a, b, c \in X$ and all $t>0$, where $g:(0, \infty) \rightarrow(0, \infty)$ is a continuous function. Then $P_{S}$ is a parametric $S$-metric and the pair $\left(X, P_{S}\right)$ is a parametric $S$-metric space.

We prove the following lemma which can be considered as the symmetry condition in a parametric $S$-metric space.

Lemma 9. Let $\left(X, P_{S}\right)$ be a parametric $S$-metric space. Then we have

$$
P_{S}(a, a, b, t)=P_{S}(b, b, a, t),
$$

for each $a, b \in X$ and all $t>0$.

Proof. Using the condition (PS2), we obtain

$$
\begin{aligned}
P_{S}(a, a, b, t) & \leq 2 P_{S}(a, a, a, t)+P_{S}(b, b, a, t) \\
& =P_{S}(b, b, a, t), \\
P_{S}(b, b, a, t) & \leq 2 P_{S}(b, b, b, t)+P_{S}(a, a, b, t) \\
& =P_{S}(a, a, b, t) .
\end{aligned}
$$


From inequalities (8), we have

$$
P_{S}(a, a, b, t)=P_{S}(b, b, a, t) .
$$

Now we give the relationship between a parametric metric and a parametric $S$-metric in the following lemma.

Lemma 10. Let $(X, P)$ be a parametric metric space and let the function $P_{S}^{P}: X \times X \times X \times(0, \infty) \rightarrow[0, \infty)$ be defined by

$$
P_{S}^{P}(a, b, c, t)=P(a, c, t)+P(b, c, t),
$$

for each $a, b, c \in X$ and all $t>0$. Then $P_{S}^{P}$ is a parametric $S$-metric and the pair $\left(X, P_{S}^{P}\right)$ is a parametric S-metric space.

Proof. It can be easily seen from Definitions 2 and 5 .

We call the parametric metric $P_{S}^{P}$ as the parametric $S$ metric generated by $P$. Notice that there exist parametric $S$ metrics $P_{S}$ satisfying $P_{S} \neq P_{S}^{P}$ for all parametric metrics. We give some examples.

Example 11. Let $X=\mathbb{R}$ and let the function $P_{S}: X \times X \times X \times$ $(0, \infty) \rightarrow[0, \infty)$ be defined by

$$
P_{S}(a, b, c, t)=t(|a-c|+|a+c-2 b|),
$$

for each $a, b, c \in \mathbb{R}$ and all $t>0$. Then $P_{S}$ is a parametric $S$ metric and the pair $\left(\mathbb{R}, P_{S}\right)$ is a parametric $S$-metric space. We have $P_{S} \neq P_{S}^{P}$; that is, $P_{S}$ is not generated by any parametric metric $P$.

Example 12. Let $X=\{f \mid f:(0, \infty) \rightarrow \mathbb{R}$ be a function $\}$ and let the function $P_{S}: X \times X \times X \times(0, \infty) \rightarrow[0, \infty)$ be defined by

$$
P_{S}(f, g, h, t)=\left|e^{f(t)}-e^{h(t)}\right|+\left|e^{f(t)}+e^{h(t)}-2 e^{g(t)}\right|,
$$

for each $f, g, h \in X$ and all $t>0$. Then $P_{S}$ is a parametric $S$ metric and the pair $\left(X, P_{S}\right)$ is a parametric $S$-metric space. We have $P_{S} \neq P_{S}^{P}$; that is, $P_{S}$ is not generated by any parametric metric $P$.

In the following lemma, we see the relationship between a parametric $b$-metric and a parametric $S$-metric.

Lemma 13. Let $\left(X, P_{S}\right)$ be a parametric $S$-metric space and let the function $P: X \times X \times(0, \infty) \rightarrow[0, \infty)$ be defined by

$$
P(a, b, t)=P_{S}(a, a, b, t),
$$

for each $a, b \in X$ and all $t>0$. Then $P$ is a parametric $b$-metric and the pair $(X, P)$ is a parametric $b$-metric space.
Proof. Using condition ( $P S 1)$, we see that conditions $(P b 1)$ and $(P b 2)$ are satisfied. Now we show that condition $(P b 3)$ is satisfied. Using condition (PS2) and Lemma 9, we have

$$
\begin{aligned}
P(a, x, t) & =P_{S}(a, a, x, t) \\
& \leq 2 P_{S}(a, a, b, t)+P_{S}(x, x, b, t) \\
& =2 P(a, b, t)+P(b, x, t), \\
P(a, x, t) & =P_{S}(x, x, a, t) \\
& \leq 2 P_{S}(x, x, b, t)+P_{S}(a, a, b, t) \\
& =P(a, b, t)+2 P(b, x, t),
\end{aligned}
$$

which implies that

$$
P(a, x, t) \leq \frac{3}{2}[P(a, b, t)+P(b, x, t)] .
$$

Then $P$ is a parametric $b$-metric with $s=3 / 2$.

Remark 14. Notice that the minimum value of $s$ is $3 / 2$. So it should be $s \neq 1$; that is, $P$ does not define a parametric metric in Lemma 13.

Definition 15. Let $\left(X, P_{S}\right)$ be a parametric $S$-metric space and let $\left\{a_{n}\right\}$ be a sequence in $X$ :

(1) $\left\{a_{n}\right\}$ converges to $x$ if and only if there exists $n_{0} \in \mathbb{N}$ such that

$$
P_{S}\left(a_{n}, a_{n}, x, t\right)<\varepsilon,
$$

for all $n \geq n_{0}$ and all $t>0$; that is,

$$
\lim _{n \rightarrow \infty} P_{S}\left(a_{n}, a_{n}, x, t\right)=0 .
$$

It is denoted by $\lim _{n \rightarrow \infty} a_{n}=x$.

(2) $\left\{a_{n}\right\}$ is called a Cauchy sequence if, for all $t>0$,

$$
\lim _{n, m \rightarrow \infty} P_{S}\left(a_{n}, a_{n}, a_{m}, t\right)=0 .
$$

(3) $\left(X, P_{S}\right)$ is called complete if every Cauchy sequence is convergent.

Lemma 16. Let $\left(X, P_{S}\right)$ be a parametric $S$-metric space. If $\left\{a_{n}\right\}$ converges to $x$, then $x$ is unique.

Proof. Let $\lim _{n \rightarrow \infty} a_{n}=x$ and let $\lim _{n \rightarrow \infty} a_{n}=y$ with $x \neq y$. Then there exists $n_{1}, n_{2} \in \mathbb{N}$ such that

$$
\begin{aligned}
& P_{S}\left(a_{n}, a_{n}, x, t\right)<\frac{\varepsilon}{4}, \\
& P_{S}\left(a_{n}, a_{n}, y, t\right)<\frac{\varepsilon}{2},
\end{aligned}
$$

for each $\varepsilon>0$, all $t>0$, and $n \geq n_{1}, n_{2}$. If we take $n_{0}=$ $\max \left\{n_{1}, n_{2}\right\}$, then, using condition (PS2) and Lemma 9, we get

$$
\begin{aligned}
P_{S}(x, x, y, t) & \leq 2 P_{S}\left(x, x, a_{n}, t\right)+P_{S}\left(y, y, a_{n}, t\right) \\
& <\frac{\varepsilon}{2}+\frac{\varepsilon}{2}=\varepsilon,
\end{aligned}
$$

for each $n \geq n_{0}$. Therefore $P_{S}(x, x, y, t)=0$ and $x=y$. 
Lemma 17. Let $\left(X, P_{S}\right)$ be a parametric $S$-metric space. If $\left\{a_{n}\right\}$ converges to $x$, then $\left\{a_{n}\right\}$ is Cauchy.

Proof. By the similar arguments used in the proof of Lemma 16, we can easily see that $\left\{a_{n}\right\}$ is a Cauchy sequence.

As a consequence of Lemma 10 and Definition 15, we obtain the following corollary.

Corollary 18. Let $(X, P)$ be a parametric metric space and let $\left(X, P_{S}^{P}\right)$ be a parametric $S$-metric space, where $P_{S}^{P}$ is generated by parametric metric $P$. Then we have the following:

(1) $\left\{a_{n}\right\} \rightarrow x$ in $(X, P)$ if and only if $\left\{a_{n}\right\} \rightarrow x$ in $\left(X, P_{S}^{P}\right)$.

(2) $\left\{a_{n}\right\}$ is Cauchy in $(X, P)$ if and only if $\left\{a_{n}\right\}$ is Cauchy in $\left(X, P_{S}^{P}\right)$.

(3) $(X, P)$ is complete if and only if $\left(X, P_{S}^{P}\right)$ is complete.

Definition 19. Let $\left(X, P_{S}\right)$ be a parametric $S$-metric space and let $T: X \rightarrow X$ be a self-mapping of $X . T$ is said to be a continuous mapping at $x$ in $X$ if

$$
\lim _{n \rightarrow \infty} P_{S}\left(T a_{n}, T a_{n}, T x, t\right)=0,
$$

for any sequence $\left\{a_{n}\right\}$ in $X$ and all $t>0$ such that

$$
\lim _{n \rightarrow \infty} P_{S}\left(a_{n}, a_{n}, x, t\right)=0 .
$$

\section{Some Fixed-Point Results}

In this section, we give some fixed-point results for expansive mappings in a complete parametric $S$-metric space.

Definition 20. Let $\left(X, P_{S}\right)$ be a parametric $S$-metric space and let $T$ be a self-mapping of $X$.

(SP1) There exist real numbers $k_{i}(i \in\{1,2,3\})$ satisfying $k_{\mathrm{i}} \geq 0(i \in\{2,3\})$ and $k_{1}>1$ such that

$$
\begin{aligned}
P_{S}(T a, T a, T b, t) \geq & k_{1} P_{S}(a, a, b, t) \\
& +k_{2} P_{S}(T a, T a, a, t) \\
& +k_{3} P_{S}(T b, T b, b, t),
\end{aligned}
$$

for each $a, b \in X$ and all $t>0$.

Theorem 21. Let $\left(X, P_{S}\right)$ be a complete parametric S-metric space and let $T$ be a surjective self-mapping of $X$. If $T$ satisfies condition (SP1), then $T$ has a unique fixed point in $X$.

Proof. Using the hypothesis, it can be easily seen that $T$ is injective. Indeed, if we take $T a=T b$, then, using condition $(S P 1)$, we get

$$
\begin{aligned}
0= & P_{S}(T a, T a, T a, t) \\
\geq & k_{1} P_{S}(a, a, b, t)+k_{2} P_{S}(T a, T a, a, t) \\
& +k_{3} P_{S}(T a, T a, b, t),
\end{aligned}
$$

for all $t>0$ and so $P_{S}(a, a, b, t)=0$; that is, we have $a=b$ since $k_{1}>1$.

Let us denote the inverse mapping of $T$ by $F$. Let $a_{0} \in X$ and define the sequence $\left\{a_{n}\right\}$ as follows:

$$
\begin{aligned}
& a_{1}=F a_{0}, \\
& a_{2}=F a_{1}=F^{2} a_{0}, \ldots, a_{n+1}=F a_{n}=F^{n+1} a_{0}, \ldots
\end{aligned}
$$

Suppose that $a_{n} \neq a_{n+1}$ for all $n$. Using condition (SP1) and Lemma 9, we have

$$
\begin{aligned}
& P_{S}\left(a_{n-1}, a_{n-1}, a_{n}, t\right) \\
&=P_{S}\left(T T^{-1} a_{n-1}, T T^{-1} a_{n-1}, T T^{-1} a_{n}, t\right) \\
& \geq k_{1} P_{S}\left(T^{-1} a_{n-1}, T^{-1} a_{n-1}, T^{-1} a_{n}, t\right) \\
& \quad+k_{2} P_{S}\left(T T^{-1} a_{n-1}, T T^{-1} a_{n-1}, T^{-1} a_{n-1}, t\right) \\
& \quad+k_{3} P_{S}\left(T T^{-1} a_{n}, T T^{-1} a_{n}, T^{-1} a_{n}, t\right) \\
&=k_{1} P_{S}\left(F a_{n-1}, F a_{n-1}, F a_{n}, t\right) \\
& \quad+k_{2} P_{S}\left(a_{n-1}, a_{n-1}, F a_{n-1}, t\right) \\
& \quad+k_{3} P_{S}\left(a_{n}, a_{n}, F a_{n}, t\right) \\
&=k_{1} P_{S}\left(a_{n}, a_{n}, a_{n+1}\right)+k_{2} P_{S}\left(a_{n-1}, a_{n-1}, a_{n}, t\right) \\
&+k_{3} P_{S}\left(a_{n}, a_{n}, a_{n+1}, t\right) \\
&=\left(k_{1}+k_{3}\right) P_{S}\left(a_{n}, a_{n}, a_{n+1}, t\right) \\
&+k_{2} P_{S}\left(a_{n-1}, a_{n-1}, a_{n}, t\right),
\end{aligned}
$$

which implies that

$$
\begin{aligned}
& \left(1-k_{2}\right) P_{S}\left(a_{n-1}, a_{n-1}, a_{n}, t\right) \\
& \quad \geq\left(k_{1}+k_{3}\right) P_{S}\left(a_{n}, a_{n}, a_{n+1}, t\right) .
\end{aligned}
$$

Clearly, we have $k_{1}+k_{3} \neq 0$. Hence, we obtain

$$
P_{S}\left(a_{n}, a_{n}, a_{n+1}, t\right) \leq \frac{1-k_{2}}{k_{1}+k_{3}} P_{S}\left(a_{n-1}, a_{n-1}, a_{n}, t\right) .
$$

If we put $k=\left(1-k_{2}\right) /\left(k_{1}+k_{3}\right)$, then we get $k<1$, since $k_{1}+k_{2}+k_{3}>1$. Repeating this process in condition (28), we find

$$
P_{S}\left(a_{n}, a_{n}, a_{n+1}, t\right) \leq k^{n} P_{S}\left(a_{0}, a_{0}, a_{1}, t\right),
$$

for all $t>0$.

Let $m, n \in \mathbb{N}$ with $m>n \geq 1$. Using inequality (29) and condition (PS2), we have

$$
P_{S}\left(a_{n}, a_{n}, a_{m}, t\right) \leq \frac{2 k^{n}}{1-k} P_{S}\left(a_{0}, a_{0}, a_{1}, t\right) .
$$

If we take limit for $n, m \rightarrow \infty$, we obtain

$$
\lim _{n, m \rightarrow \infty} P_{S}\left(a_{n}, a_{n}, a_{m}, t\right)=0 .
$$


Therefore $\left\{a_{n}\right\}$ is Cauchy. Then there exists $y \in X$ such that

$$
\lim _{n \rightarrow \infty} a_{n}=y,
$$

since $\left(X, P_{S}\right)$ is a complete parametric $S$-metric space. Using the surjectivity hypothesis, there exists a point $x \in X$ such that $T x=y$. From condition $(S P 1)$, we have

$$
\begin{aligned}
P_{S}\left(a_{n}, a_{n}, y, t\right)= & P_{S}\left(T a_{n+1}, T a_{n+1}, T x, t\right) \\
\geq & k_{1} P_{S}\left(a_{n+1}, a_{n+1}, x, t\right) \\
& +k_{2} P_{S}\left(a_{n}, a_{n}, a_{n+1}, t\right) \\
& +k_{3} P_{S}(y, y, x, t) .
\end{aligned}
$$

If we take limit for $n \rightarrow \infty$, we obtain

$$
0 \geq\left(k_{1}+k_{3}\right) P_{S}(y, y, x, t),
$$

which implies that $y=x$ and $T y=y$.

Now we show the uniqueness of $y$. Let $z$ be another fixed point of $T$ with $y \neq z$. Using condition (SP1) and Lemma 9, we get

$$
\begin{aligned}
P_{S}(y, y, z, t)= & P_{S}(T y, T y, T z, t) \\
\geq & k_{1} P_{S}(y, y, z, t)+k_{2} P_{S}(y, y, y, t) \\
& +k_{3} P_{S}(z, z, z, t)=k_{1} P_{S}(y, y, z, t),
\end{aligned}
$$

which implies that $y=z$, since $k_{1}>1$. Consequently, $T$ has a unique fixed point $y$.

We give some examples which satisfy the conditions of Theorem 21 .

Example 22. Let $X=\mathbb{R}^{+} \cup\{0\}$ be the complete $S$-metric space with the $S$-metric defined in Example 8. Let us define the selfmapping $T: \mathbb{R}^{+} \cup\{0\} \rightarrow \mathbb{R}^{+} \cup\{0\}$ as

$$
T x=\beta x,
$$

for all $x \in \mathbb{R}$ with $\beta>1$, and the function $g:(0, \infty) \rightarrow$ $(0, \infty)$ as

$$
g(t)=t^{2}
$$

for all $t \in(0, \infty)$. Then $T$ satisfies the conditions of Theorem 21 with $k_{1}=\beta$ and $k_{2}=k_{3}=0$. Then $T$ has a unique fixed point $x=0$ in $X$.

Example 23. Let $X=\mathbb{R}^{+} \cup\{0\}$ be the complete $S$-metric space with the $S$-metric defined in Example 8. Let us define the selfmapping $T: \mathbb{R}^{+} \cup\{0\} \rightarrow \mathbb{R}^{+} \cup\{0\}$ as

$$
T x=x+\ln (x+1),
$$

for all $x \in \mathbb{R}$ with $\beta>1$, and the function $g:(0, \infty) \rightarrow$ $(0, \infty)$ as

$$
g(t)=t^{3}+t^{2}+t+1,
$$

for all $t \in(0, \infty)$. Then $T$ satisfies the conditions of Theorem 21 with $k_{1}=\min \{\ln (x+1) / x: x \neq 0 \in X\}$ and $k_{2}=k_{3}=0$. Then $T$ has a unique fixed point $x=0$ in $X$.
If we take $k_{2}=k_{3}$ in condition (SP1), then we obtain the following corollary.

Corollary 24. Let $\left(X, P_{S}\right)$ be a complete parametric S-metric space and let $T$ be a surjective self-mapping of $X$. If there exist real numbers $k_{i}(i \in\{1,2\})$ satisfying $k_{1}>1$ and $k_{2} \geq 0$ such that

$$
\begin{aligned}
P_{S}(T a, T a, T b, t) \\
\geq k_{1} P_{S}(a, a, b, t) \\
\quad+k_{2} \max \left\{P_{S}(T a, T a, a, t), P_{S}(T b, T b, b, t)\right\},
\end{aligned}
$$

for each $a, b \in X$ and all $t>0$, then $T$ has a unique fixed point in $X$.

If we take $k_{1}=k$ and $k_{2}=k_{3}=0$ and $k_{1}=k$ and $k_{2}=0$ in Theorem 21 and Corollary 24, respectively, then we obtain the following corollaries.

Corollary 25. Let $\left(X, P_{S}\right)$ be a complete parametric $S$-metric space and let $T$ be a surjective self-mapping of $X$. If there exists a real number $k>1$ such that

$$
P_{S}(T a, T a, T b, t) \geq k P_{S}(a, a, b, t),
$$

for each $a, b \in X$ and all $t>0$, then $T$ has a unique fixed point in $X$.

Corollary 26. Let $\left(X, P_{\mathrm{S}}\right)$ be a complete parametric S-metric space and let $T$ be a surjective self-mapping of $X$. If there exist a positive integer $m$ and a real number $k>1$ such that

$$
P_{S}\left(T^{m} a, T^{m} a, T^{m} b, t\right) \geq k P_{S}(a, a, b, t),
$$

for each $a, b \in X$ and all $t>0$, then $T$ has a unique fixed point in $X$.

Proof. From Corollary 25, by a similar way used in the proof of Theorem 21, it can be easily seen that $T^{m}$ has a unique fixed point $a$ in $X$. Also we have

$$
T a=T T^{m} a=T^{m+1} a=T^{m} T a
$$

and so we obtain that $T a$ is a fixed point for $T^{m}$. We get $T a=a$, since $a$ is the unique fixed point.

\section{Competing Interests}

The authors declare that there are no competing interests regarding the publication of this paper.

\section{References}

[1] S. Z. Wang, B. Y. Li, Z. M. Gao, and K. Iseki, "Some fixed point theorems for expansive mappings," Mathematica Japonica, vol. 29, pp. 631-636, 1984.

[2] S. Sedghi, N. Shobe, and A. Aliouche, "A generalization of fixed point theorems in S-metric spaces," Matematički Vesnik, vol. 64, no. 3, pp. 258-266, 2012. 
[3] N. T. Hieu, N. T. Thanh Ly, and N. V. Dung, "A generalization of ciric quasi-contractions for maps on S-metric spaces," Thai Journal of Mathematics, vol. 13, no. 2, pp. 369-380, 2015.

[4] N. Y. Özgür and N. Taş, "Some generalizations of fixed point theorems on S-metric spaces," in Essays in Mathematics and Its Applications: In Honor of Vladimir Arnold, Springer, New York, NY, USA, 2016.

[5] N. Y. Özgür and N. Taş, "Some fixed point theorems on S-metric spaces," Matematički Vesnik, In press.

[6] S. Sedghi and N. V. Dung, "Fixed point theorems on S-metric spaces," Matematichki Vesnik, vol. 66, no. 1, pp. 113-124, 2014.

[7] N. Hussain, S. Khaleghizadeh, P. Salimi, and A. A. N. Abdou, "A new approach to fixed point results in triangular intuitionistic fuzzy metric spaces," Abstract and Applied Analysis, vol. 2014, Article ID 690139, 16 pages, 2014.

[8] N. Hussain, P. Salimi, and V. Parvaneh, "Fixed point results for various contractions in parametric and fuzzy b-metric spaces," Journal of Nonlinear Science and Its Applications, vol. 8, no. 5, pp. 719-739, 2015.

[9] K. P. R. Rao, D. V. Babu, and E. T. Ramudu, "Some unique common fixed point theorems in parametric s-metric spaces," International Journal of Innovative Research in Science, Engineering and Technology, vol. 3, no. 7, pp. 14375-14387, 2014.

[10] R. Jain, R. D. Daheriya, and M. Ughade, "Fixed point, coincidence point and common fixed point theorems under various expansive conditions in parametric metric spaces and parametric b-metric spaces," Gazi University Journal of Science, vol. 29, no. 1, pp. 95-107, 2016. 


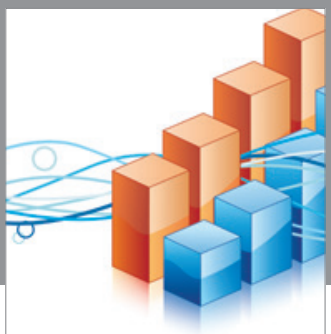

Advances in

Operations Research

vatem alat4

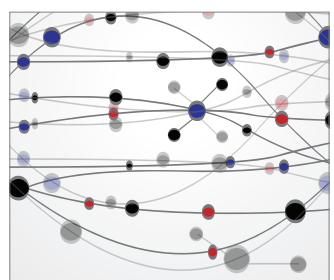

\section{The Scientific} World Journal
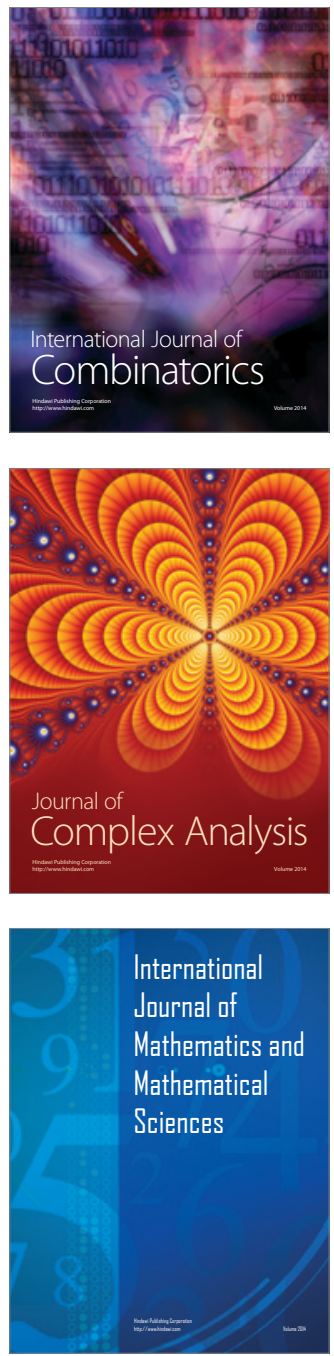
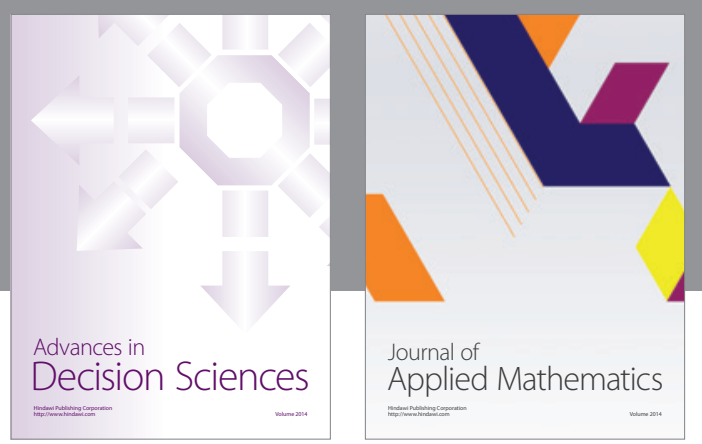

Algebra

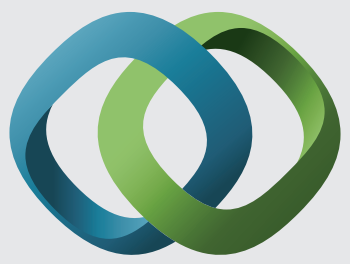

\section{Hindawi}

Submit your manuscripts at

http://www.hindawi.com
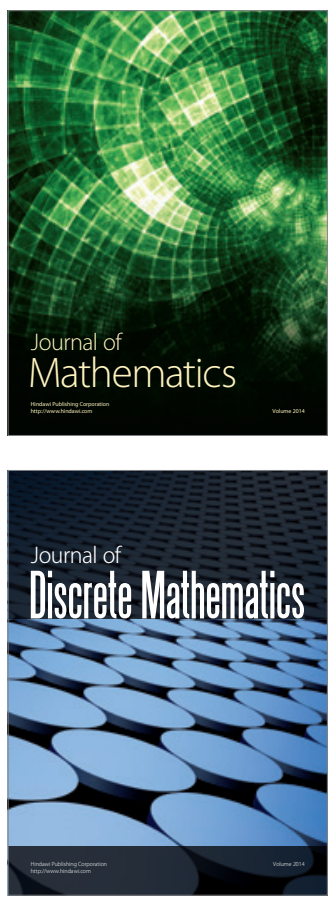

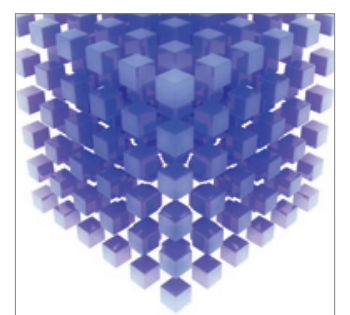

Mathematical Problems in Engineering
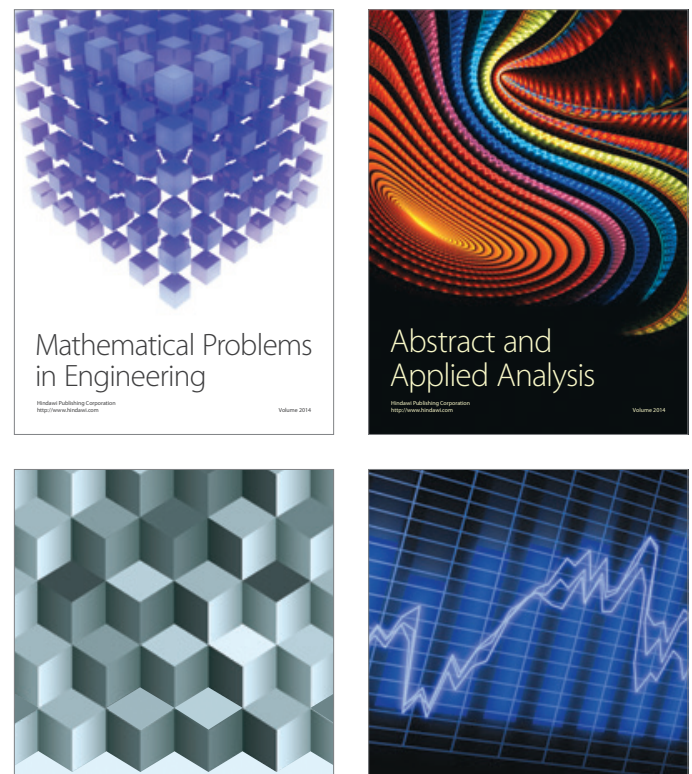

Journal of

Function Spaces

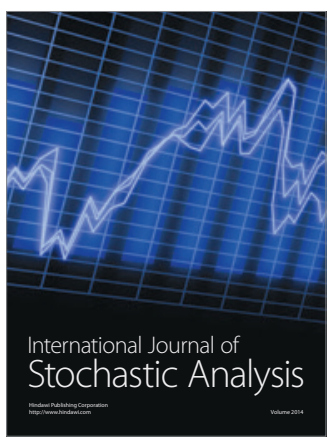

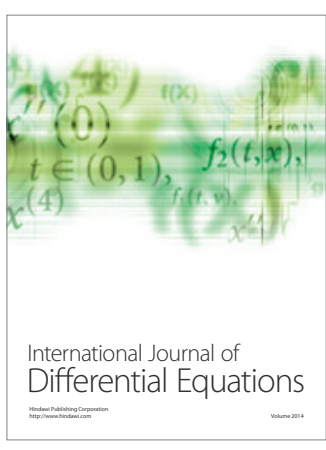
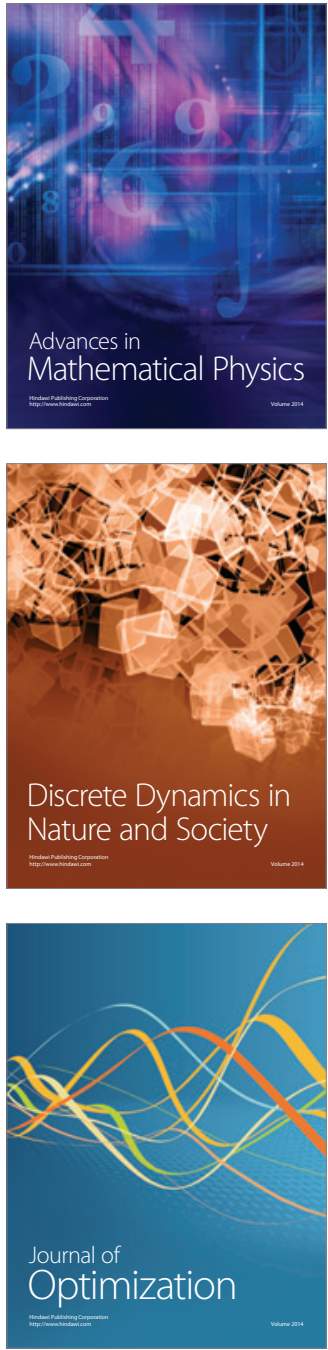